\title{
Alstroemeria hookeri Lodd. subsp. sansebastiana C.M.Baeza \& E.Ruiz, nueva para la flora de Chile
}

\author{
Alstroemeria hookeri Lodd. subsp. sansebastiana C.M.Baeza \& E.Ruiz, new for the \\ Chilean flora
}

\author{
Carlos M. Baeza \& Eduardo Ruiz \\ Departamento de Botánica, Universidad de Concepción, Casilla 160-C, Concepción, Chile \\ cbaeza@udec.cl
}

\begin{abstract}
We describe a new subspecies of Alstroemeria hookeri in Chile. This new species grows in the central Valley of the Region of Bío-Bío, Chile. It differs from the typical subspecies by its larger size, lighter-colored flowers and different geographical distribution.
\end{abstract}

Alstroemeria L. (Alstroemeriaceae) es un género americano, que comprende alrededor de 49 taxones, distribuidos principalmente en Brasil, Argentina y Chile, ocupando una variada gama de ambientes y que crecen desde el nivel del mar hasta sobre los $3.700 \mathrm{~m}$ de altitud en Chile (Muñoz \& Moreira 2003). Chilecentral constituye el centro de diversidad de este género (Bayer 1987) con una distribución disyunta también importante en el este y centro de Brasil (Aker \& Healy 1990). En Chile, Alstroemeria está representado por 31-33 especies (Bayer 1987, Muñoz \& Moreira 2003) distribuidas desde el extremo norte hasta la Patagonia, siendo la zona central la que concentra la mayor cantidad de especies (Muñoz \& Moreira 2003). Alstroemeria es uno los géneros de la flora vascular chilena más diversificados y con una gama de colores y tamaños de sus flores que hacen que las especies de este género presenten un alto potencial económico como flores de corte y de cultivo, lo que se puede observar en el comercio. Debido a la importancia económica que está alcanzando el género, es fundamental tener clara la taxonomía de este grupo de plantas en Chile, aspecto que en general está bien resuelto con el trabajo taxonómico de Bayer (1987) y las posteriores contribuciones hechas por Muñoz (2000) y Muñoz \& Moreira (2003). Sin embargo, aún persisten algunos problemas taxonómicos en complejos donde los límites específicos no están claros y las entidades se describen como subespecies. Esto podría deberse a los activos procesos microevolutivos que pueden estar experimentando las especies (Ruiz et al. 2010). Uno de estos complejos está constituido por las 4 subespecies de $A$. hookeri Lodd.: A. hookeri subsp. hookeri, A. hookeri subsp. recumbens (Herbert) Bayer, A. hookeri subsp. maculata Bayer y A. hookeri subsp. cummingiana (Herbert) Bayer
(Bayer 1987). Por otro lado, Muñoz \& Moreira (2003) sólo reconocen las tres primeras subespecies, manteniendo a $A$. cummingiana Herbert a nivel específico.

Durante el desarrollo del proyecto Fondecyt No 1070520 se realizaron recolecciones de material de Alstroemeria pertenecientes al complejo $A$. hookeri Lodd. a lo largo de toda su distribución $\left(31^{\circ}-37^{\circ} \mathrm{S}\right)$. En la Región del Bío-Bío (VIII) se recolectaron 19 poblaciones de $A$. hookeri Lodd. subsp. hookeri, 11 se hicieron en el rango costero de su distribución y 8 en la zona del valle central (Pangal del Laja, ver mapa de distribución en Cajas et al. 2009). Luego de un detallado y minucioso análisis comparativo entre estas poblaciones costeras y las del valle central, separadas por la Cordillera de la Costa (Nahuelbuta) se concluyó que las poblaciones del interior corresponden a una nueva entidad taxonómica para Chile que aquí se presenta. En este trabajo se describe Alstroemeria hookeri subsp. sansebastiana C.M. Baeza \& E. Ruiz, se indica su distribución geográfica y su ecología.

Alstroemeria hookeri Lodd. subsp. sansebastiana C.M. Baeza \& E. Ruiz, subsp. nov.

TIPO: Chile. VIII Región. Provincia de Bío-Bío. Yumbel. Camino estación Yumbel hacia Puente Perales, $102 \mathrm{~m}$, (3709'S-72³2'W). 30-XII-2002. C. Baeza 4187 (CONC). (Holotipo, CONC). Fig. 1 A.

Planta perennis, brevis, 11,8-48,2 cm alta, erecta. Radix cylindrica. Caulis glaber. Folia alterna, glabra, 1,5-4,7 cm longa et 2-5 mm lata, lanceolata. Tepala exteriora roseotenuis; interiora duo superiora oblanceolata, 2,7-3,6 cm lata, rosea tenuis ad intensibus, lineis luteus, aureus et 
distincte purpureo variegata; interiori-inferius 2,5-3,9 cm longa et 0,7-1 cm lata. Antherae 2-7 mm longa.

Hierbas perennes, con rizomas cilíndricos, simples; tallos erectos, raro decumbentes, de 11,8-48,2 cm de long., hojas de $1,5-4,7 \mathrm{~cm}$ de largo y $0,2-0,5 \mathrm{~cm}$ de ancho, angostamente lanceoladas, algo ensanchadas en la base, alternas, sésiles, de borde liso. Inflorescencias cimosas, umbeliformes, de 5-10 cm, 3-13-floras, con distintos grados de ramificación, divididas en varios ejes, que a su vez vuelven a dividirse, y portan 3-5-flores en cada uno, brácteas florales similares a las hojas, de 1-2,3 cm de long. Flores zigomorfas, 3,3$4,9 \mathrm{~cm}$, con perigonio corolino, pedicelos de $1,3-2 \mathrm{~cm}$ de long. Tépalos 6, los 3 externos de 3,1-4,3 cm de long. $\mathrm{x}$ 0,76-1,46 cm de ancho, de color rosa pálido, con 5, 6 ó 7 nervios conspicuos, mucrón de 4,2-6,1 mm, de color verde, tépalos uniformes, sin guías. De los tépalos internos, los 2 superiores generalmente más angostos, de 2,7-3,6 de long. x 0,7-1 cm de ancho, oblanceolados, trinerves, de color rosa pálido, con una conspicua franja amarilla que hacia la base varía de rosa pálido a blanquecino o blanco, con un patrón de manchas de cortas líneas color marrón, las más notorias dispuestas en la franja amarilla. Nectarios de forma tubular en la base, en el tercio inferior, el cual se pliega sin fusionarse, de 2,1-6,1 $\mathrm{mm}$ de long. El tépalo interno inferior romboidal a oblanceolado, de 2,5-3,9 $\mathrm{cm}$ long. x 0,7-1 cm, de color rosa pálido, sin la franja amarilla, con 3-nervios conspicuos, levemente más ancho y corto que los dos tépalos internos superiores, abierto en la base del tépalo. En todos los tépalos internos hay papilas en el borde exterior e interior. Androceo hexámero, anteras principalmente de tamaño regular en cada flor, de 2-7 mm de largo x 1-1,8 $\mathrm{mm}$ de ancho, filamentos de 2,5$2,9 \mathrm{~cm}$. Ovario ínfero, sincarpelar, con numerosos óvulos, trilocular, de 0,5 0,7 cm long. x 0,4-0,6 cm ancho, con 6 costillas, estilo linear, de 2-2,9 cm long., blanquecino hacia la base y fucsia hacia el ápice, estigma trífido, linear, de color rosa claro con ramas de 2-4 mm long. Cápsulas globosas, con nervios prominentes, de 1,5-2 cm de long., con dehiscencia explosiva. Semillas numerosas, de color castaño oscuro, redondeadas.

Etimología: Esta nueva subespecie está dedicada a San Sebastián, Santo Patrón de Yumbel, insigne mártir de la iglesia católica, cuya imagen en madera de cedro, traída por los españoles en 1655, es venerada cada año en esta ciudad.

Distribución y eCología: Esta nueva subespecie crece en suelos arenosos, muy secos en el Pangal del Laja, VIII Región (Baeza et al. 2010). Ocupa una de las zonas del país con mayor grado de erosión y perturbación humana, donde su hábitat ha sido ocupado sustancialmente por plantaciones de Pinus radiata D.Don (pino de Monterrey). Esta condición de crecer en hábitats totalmente fragmentados, con una extensión de la presencia estimada de $400 \mathrm{~km}^{2}$, sin representación en algún área protegida o por proteger, permiten considerar esta subespecie como en la categoría En Peligro (EN B1ab(iii)).
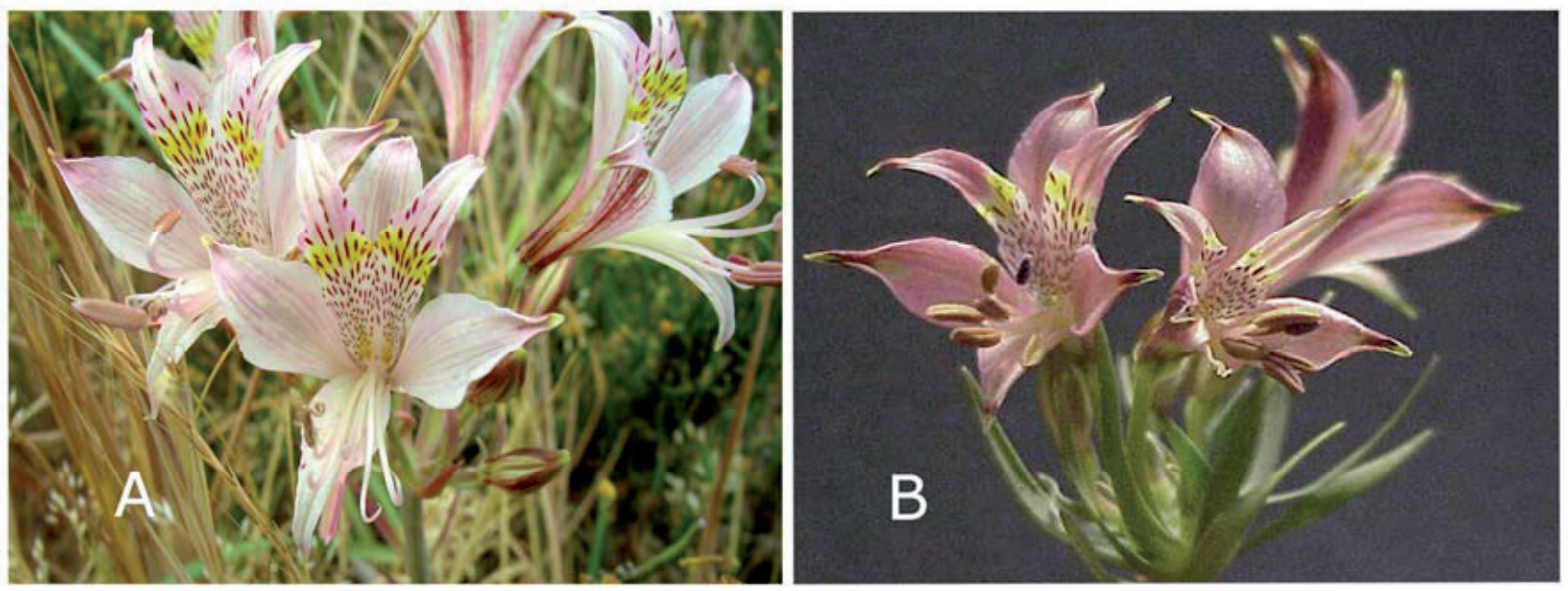

Figura 1. Se muestra una planta con flores de A. hookeri subsp. sansebastiana (A) y de A. hookeri subsp. hookeri (B). Se observan las diferencias en la coloración, la ornamentación y en el tamaño de las inflorescencias.

Figure. 1. An individual of $A$. hookeri subsp. sansebastiana (A) and one of $A$. hookeri subsp. hookeri (B). It can be seen the differentiation of coloration, ornamentation and size of the inflorescence. 
Material adicional estudiado: CHILE, Región del BíoBío, Prov. Bío-Bío, camino de Yumbel hacia la carretera 5 Sur, 100 m (3708'S-72²7'W), 7-XII-2004. C. Baeza 4212 (CONC); camino de Laja hacia la carretera 5 Sur, $100 \mathrm{~m}$ (37²13'S-72³0'W), 7-XII-2004. C. Baeza 4214 (CONC); Yumbel, salida sur, $100 \mathrm{~m}\left(37^{\circ} 08^{\prime} \mathrm{S}-72^{\circ} 32^{\prime} \mathrm{W}\right), 7-X I I-2004$. C. Baeza 4215 (CONC); camino entre Yumbel y Cabrero, $2 \mathrm{~km}$ al este del puente Ibáñez, 100 m (3700'S-72²9’W), 7-XII-2004. C. Baeza 4216 (CONC); camino de Cabrero a Bulnes, 3,5 km desde Cabrero, orilla ruta 5 sur, $100 \mathrm{~m}$ (37001'S-72²1'W), 7-XII-2004. C. Baeza 4217 (CONC). Prov. Nuble, camino hacia Quillón, sector Los Alemanes, $100 \mathrm{~m}\left(36^{\circ} 46^{\prime} \mathrm{S}-72^{\circ} 24^{\prime} \mathrm{W}\right), 7-X I I-2004$. C. Baeza 4218 (CONC); entrada a Quillón, casi al frente de la laguna Avendaño, 70 m (36²4'S-72²7'W), 7-XII-2004. C. Baeza 4219 (CONC); puente El Roble, 64 m (36 $\left.{ }^{\circ} 45^{\prime} \mathrm{S}-72^{\circ} 25^{\prime} \mathrm{W}\right)$, 12-XII-2002. C. Baeza 4175 (CONC).

Esta nueva subespecie puede separarse de $A$. hookeri subsp. hookeri por medio de la siguiente clave:

1. Plantas de tamaño pequeño, raro mayores de $20 \mathrm{~cm}$ de altura, flores de color rosado intenso, inflorescencias con pedicelos cortos, raro mayores de $3 \mathrm{~cm}$, manchas de los tépalos internos de color fucsia. Crecen en la costa de las regiones del Maule y Bío-Bío

A. hookeri subsp. hookeri

1.' Plantas mayores de $20 \mathrm{~cm}$ de altura, flores de color rosado claro, inflorescencias con pedicelos largos, raro menores de $3 \mathrm{~cm}$, manchas de los tépalos internos de color marrón. Crecen en el interior de la Región del Bío-Bío A. hookeri subsp. sansebastiana

Además de los caracteres indicados en la clave anterior, hay una serie de otros antecedentes que permiten reconocer a esta nueva subespecie entre las asignadas al complejo $A$. hookeri. Baeza et al. (2007) reconocen diferencias notables en el cariotipo y en la ubicación de genes ribosomales entre las poblaciones costeras y las del interior. Cajas et al. (2009) en un detallado estudio poblacional en $A$. hookeri subsp. hookeri, empleando las mismas poblaciones, encuentran también diferencias significativas en el cariotipo, en los índices de asimetría y en la longitud total de los cromosomas entre estas poblaciones. Trabajando con plantas de las poblaciones aludidas, Ruiz et al. (2010) a su vez dan cuenta de los resultados obtenidos en un estudio de morfología comparada, los que combinaron con los obtenidos a partir de una caracterización aloenzimática, donde se encontró que existe cierta tendencia hacia la diferenciación morfológica entre las poblaciones de la costa y del interior. Aloenzimáticamente, estas diferencias no son evidentes y los resultados indicaron que las poblaciones más diferentes genéticamente son del interior (Ruiz et al. 2010)

\section{AGRADECIMIENTOS}

Se agradece el apoyo de Fondecyt $N^{o}$ 1070520, al Departamento de Botánica de la Universidad de Concepción por las facilidades otorgadas y a los dos revisores anónimos que contribuyeron a mejorar sustancialmente el trabajo.

\section{BIBLIOGRAFÍA}

Aker, S. \& Healy, W. 1990. The phytogeography of the genus Alstroemeria. Herbertia 46: 76-87.

Baeza, C., O. Schrader \& H. Budahn 2007. Characterization of geographically isolated accessions in five Alstroemeria L. species (Chile) using FISH of tandemly repeated DNA sequences and RAPD analysis. Plant Systematics and Evolution 269: 1-14.

Baeza, C., E. Ruiz \& M. Negritto. 2010. Comparative karyotypic analysis in the Alstroemeria hookeri Lodd. (Alstroemeriaceae) complex sensu Bayer (1987). Genetics and Molecular Biology 33(1): 119-124.

BAYER, E. 1987. Die Gattung Alstroemeria in Chile. Mitteilungen der Botanischen Staatssamlungen München 24: 1-362.

Cajas, D., Baeza, M., Ruiz, E. \& M. Negritto 2009. Análisis citogenético en poblaciones de Alstroemeria hookeri Lodd. ssp. hookeri (Alstroemeriaceae) en la Región del Bío-Bío, Chile. Gayana Botánica 66(2): 117-126.

MuÑoz, M. 2000. Novedades en la familia Alstroemeriaceae. Gayana Botánica 57: 55-59.

Muñoz, M. \& A. Moreira. 2003. Alstroemerias de Chile. Diversidad, distribución y conservación. Taller La Era, Santiago. 140 pp.

Ruiz, E., K. Balboa, M. A. Negritto, C. Baeza \& V. Briceño. 2010.Variabilidad genética y morfológica y estructuración poblacional en Alstroemeria hookeri subsp. hookeri (Alstroemeriaceae), endémica de Chile. Revista Chilena de Historia Natural 83: 605-616. 